\title{
Evolution of electroluminescence from multiple Si-implanted silicon nitride films with thermal annealing
}

\author{
Z. H. Cen, ${ }^{1, a)}$ T. P. Chen, ${ }^{1, b)}$ L. Ding, ${ }^{1}$ Y. Liu, ${ }^{1,2}$ J. I. Wong, ${ }^{1}$ M. Yang, ${ }^{1}$ Z. Liu, ${ }^{1}$ W. P. Goh, ${ }^{3}$ \\ F. R. Zhu, ${ }^{3}$ and S. Fung ${ }^{4}$ \\ ${ }^{1}$ School of Electrical and Electronic Engineering, Nanyang Technological University, Singapore 639798, \\ Singapore \\ ${ }^{2}$ State Key Laboratory of Electronic Thin Films and Integrated Devices, University of Electronic Science \\ and Technology of China, Chengdu, Sichuan, 610054, People's Republic of China \\ ${ }^{3}$ Institute of Materials Research and Engineering, Singapore 117602, Singapore \\ ${ }^{4}$ Department of Physics, The University of Hong Kong, Hong Kong Island, Hong Kong
}

(Received 28 February 2009; accepted 8 May 2009; published online 16 June 2009)

Influence of thermal annealing on electroluminescence (EL) from multiple-Si-implanted silicon nitride films has been investigated. A reduced injection current and an enhanced EL intensity have been obtained simultaneously by increasing the annealing temperature, which results in a higher EL quantum efficiency. In addition, four emission bands are identified, and their peak energies, intensities, and full widths at half maxima are found to change with annealing temperature. A model is proposed to illustrate the carrier transport, the mechanisms of the four emission bands, and the evolution of the EL bands with annealing as well. The two major bands and the minor ultraviolet band are explained in terms of the recombination of the injected electrons in either the silicon dangling-bond $\left(\equiv \mathrm{Si}^{0}\right)$ states or the nitride conduction band with the injected holes in either the band tail states above the nitride valence band or the valence band itself, while the minor near infrared band is attributed to the Si nanocrystals formed in the thin film. (C) 2009 American Institute of Physics. [DOI: 10.1063/1.3148248]

\section{INTRODUCTION}

Significant progress has been made in the research of $\mathrm{Si}$ light sources based on $\mathrm{Si}$ nanoparticles dispersed in $\mathrm{SiO}_{2}$ or Si-rich silicon oxide. ${ }^{1-3}$ However, the quantum efficiency of electrically driven light emission from these oxide-based systems is not yet sufficient for practical applications. Recently, silicon nitride thin films with excess Si have attracted great interest as a promising alternative. ${ }^{4-11}$ Compared to the oxide-based active materials, Si-rich silicon nitride (SRN) has lower barriers for carrier injections, yielding a lower threshold voltage for light emitting devices (LEDs). In addition, theoretical simulations and experiments indicate that the presence of $\mathrm{N}$ atoms bonded to the surface of Si nanoparticles enhances the probability of radiative recombination and thus the light emission efficiency. ${ }^{5}$ Moreover, the large refractive index of the SRN materials benefits their applications in photonic crystal structures such as the nanocavity that can enhance the light emission efficiency and narrow the emission band. ${ }^{6}$ Tunable photoluminescence (PL) from near infrared (IR) to violet and visible electroluminescence (EL) have been achieved from various SRN materials. ${ }^{4-11}$ In this work, SRN thin films are synthesized by multiple $\mathrm{Si}$ ion implantations into silicon nitride thin films at various energies with different doses to achieve strong visible EL, and an investigation on the effect of thermal annealing on the EL has been conducted.

\footnotetext{
${ }^{a)}$ Electronic mail: cenz0001@ntu.edu.sg.

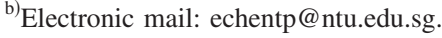

\section{EXPERIMENTAL DETAILS}

A $30 \mathrm{~nm}$ silicon nitride thin film was deposited on a $p$-type $\mathrm{Si}$ (100) wafer by low-pressure chemical vapor deposition. The silicon nitride thin film was implanted with $\mathrm{Si}$ ions at room temperature. In this study, a multiple $\mathrm{Si}$ ion implantation process was employed to obtain a uniform distribution of the implanted $\mathrm{Si}$ ions. This process has been found to be able to yield a strong visible EL. ${ }^{2,11}$ The recipe of the $\mathrm{Si}$ ion implantations is as follows: the first implantation at the energy of $25 \mathrm{keV}$ with the dose of 4 $\times 10^{16}$ atoms $/ \mathrm{cm}^{2}$; the second implantation at $8 \mathrm{keV}$ with the dose of $8 \times 10^{15}$ atoms $/ \mathrm{cm}^{2}$; and the third implantation at $2 \mathrm{keV}$ with the dose of $3 \times 10^{15}$ atoms $/ \mathrm{cm}^{2}$. From the stopping and range of ions in matter simulation, the average excess Si concentration is $\sim 1.2 \times 10^{22} \mathrm{~cm}^{-3}$ and the corresponding volume fraction of excess $\mathrm{Si}$ in the nitride layer is about $25 \%$. The Si-implanted silicon nitride film was annealed at either 800 or $1100{ }^{\circ} \mathrm{C}$ for $1 \mathrm{~h}$ in nitrogen ambient. To construct the LED structure, a 100-nm-thick transparent indium tin oxide (ITO) layer was deposited by sputtering technique to form the transparent electrode with a diameter of $1.2 \mathrm{~mm}$, and a $200 \mathrm{~nm}$ aluminum (Al) layer was deposited on the backside of Si substrate to form the back electrode by electron-beam evaporation. EL excitation was carried out under a negative gate voltage with a Keithley 2400 Source Meter. The EL spectra were measured by a PDS-1 photomultiplier tube detector together with a monochrometer. The current-voltage $(I-V)$ characteristics of the LED were measured at room temperature by a Keithley 4200 semiconductor characterization system. 


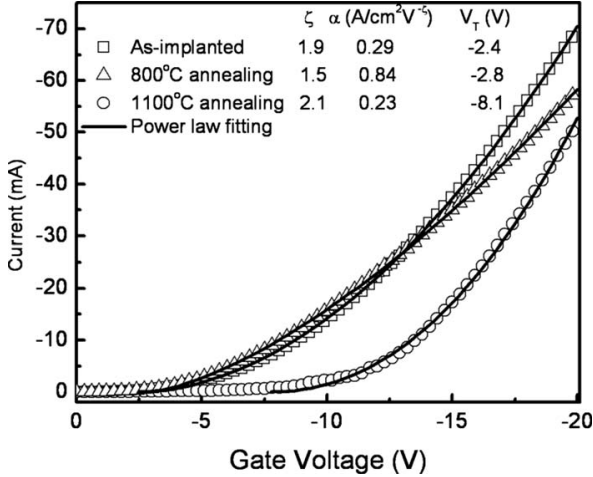

FIG. 1. I-V characteristics for the as-implanted and the annealed films. The values of the parameters of Eq. (1) obtained from the power law fitting are given in the figure.

\section{RESULTS AND DISCUSSION}

Figure 1 shows the $I-V$ characteristics for the asimplanted and annealed thin films. As can be seen in the figure, the thermal annealing at $800{ }^{\circ} \mathrm{C}$ induces a reduction in the current conduction at the gate voltages with a magnitude larger than $12 \mathrm{~V}$, as compared with the as-implanted film. The current reduction becomes more significant and in a wider voltage range for the annealing at $1100{ }^{\circ} \mathrm{C}$. The annealing-induced current reduction can be attributed to the recovery of the silicon nitride matrix during the thermal annealing process. ${ }^{12}$ As can be seen in Fig. 1 , the $I-V$ characteristics follow a power law

$$
I=\alpha_{0}\left(V-V_{T}\right)^{\zeta},
$$

where $I$ is the current, $V$ is the gate voltage, $V_{T}$ is the global threshold voltage, $\zeta$ is the scaling exponent, and $\alpha_{0}$ is a coefficient. ${ }^{13}$ The values of the parameters obtained from the power law fitting are listed in Fig. 1. The yielded $\zeta$ values are in the range from $\sim 1.5$ to $\sim 2.1$, suggesting that the current conduction is close to the two-dimensional transport. ${ }^{13}$ The power law behavior can be explained in terms of the formation of conductive percolation tunneling paths by the $\mathrm{Si}$ nanoclusters as well as the defect states created by the ion implantation. ${ }^{14,15}$ With a large number of Si nanoclusters and defect states distributed in the thin film, the injected carriers can be transported through the tunneling via the Si nanoclusters and the defect states. As the defect states can be removed by the annealing, the tunneling via the defect states will be suppressed by the annealing. ${ }^{12}$ This explains the increase in the global threshold voltage or the reduction in the conduction current with annealing temperature.

Under the negative gate voltage applied on the ITO gate, strong EL can be observed by the naked eye in darkness from the LED based on Si-implanted silicon nitride films. For both the as-implanted and annealed films, the EL spectrum does not change in shape with the gate voltage significantly. As an example, Fig. 2 shows the dependence of EL spectrum on the gate voltage for the annealing at $800{ }^{\circ} \mathrm{C}$. For both the asimplanted film and the film annealed at $800{ }^{\circ} \mathrm{C}$, the integrated EL intensity increases with the voltage, as shown in Fig. 3. In addition, there is a linear relationship between the integrated EL intensity and the injection current, as shown in the inset of Fig. 2, implying that the EL originates from the

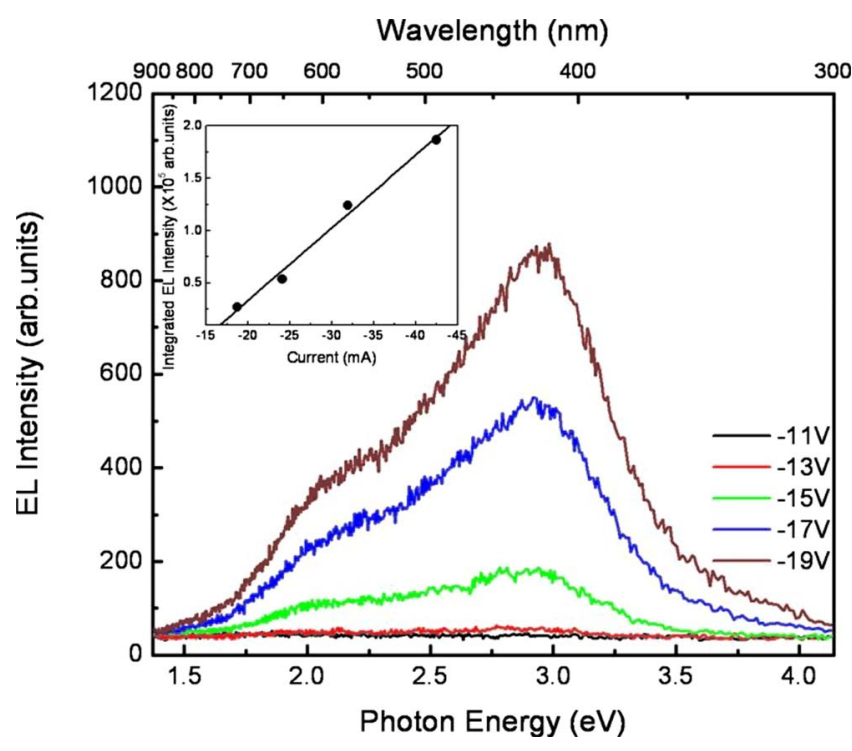

FIG. 2. (Color online) EL spectra of the $800{ }^{\circ} \mathrm{C}$ annealed film under different gate voltages. The inset shows the linear relation between the integrated EL intensity and the injection current.

recombination of electrons and holes injected from the ITO gate and the $\mathrm{Si}$ substrate, respectively. However, for the sample annealed at $1100{ }^{\circ} \mathrm{C}$, as can be seen in Fig. 3, the integrated EL intensity decreases when the voltage magnitude is larger than $\sim 16 \mathrm{~V}$. The quenching phenomenon has been attributed to the high electric field effect and the Auger recombination under high-level injection. ${ }^{11}$ On the other hand, due to a large increase in the EL intensity (Fig. 3) and a large reduction in the injection current (Fig. 1) as results of annealing, the EL quantum efficiency will be enhanced strongly by annealing. In addition, as can be seen in Fig. 3, the annealing also reduces the EL turn-on voltage significantly. Luminescence efficiency is constrained by the nonradiative recombination and the luminescence centers. When the Si-implanted film is annealed in $\mathrm{N}_{2}$, the nonradiative defects can be removed by the nitrogen passivation and the recovery of implantation damages. ${ }^{16}$ On the other hand, $\mathrm{Si}$ nanoclusters and luminescent centers could be formed during annealing, ${ }^{17,18}$ particularly at a high temperature. ${ }^{19}$ With reduction in the nonradiative defects and activation of the luminescence centers, the light emission quantum efficiency can be improved.

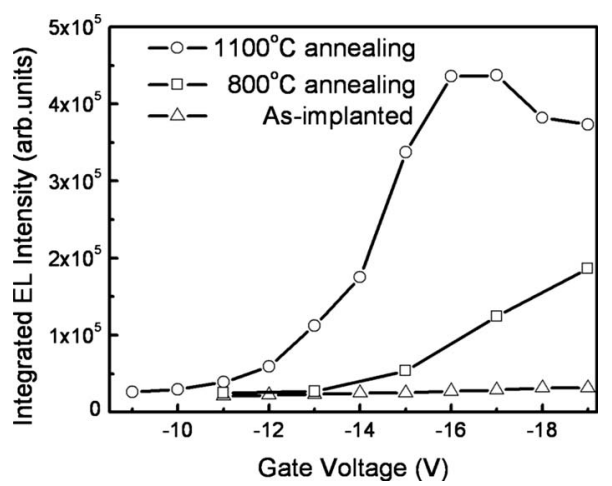

FIG. 3. Integrated EL intensity for the as-implanted and annealed films as a function of the gate voltages. 


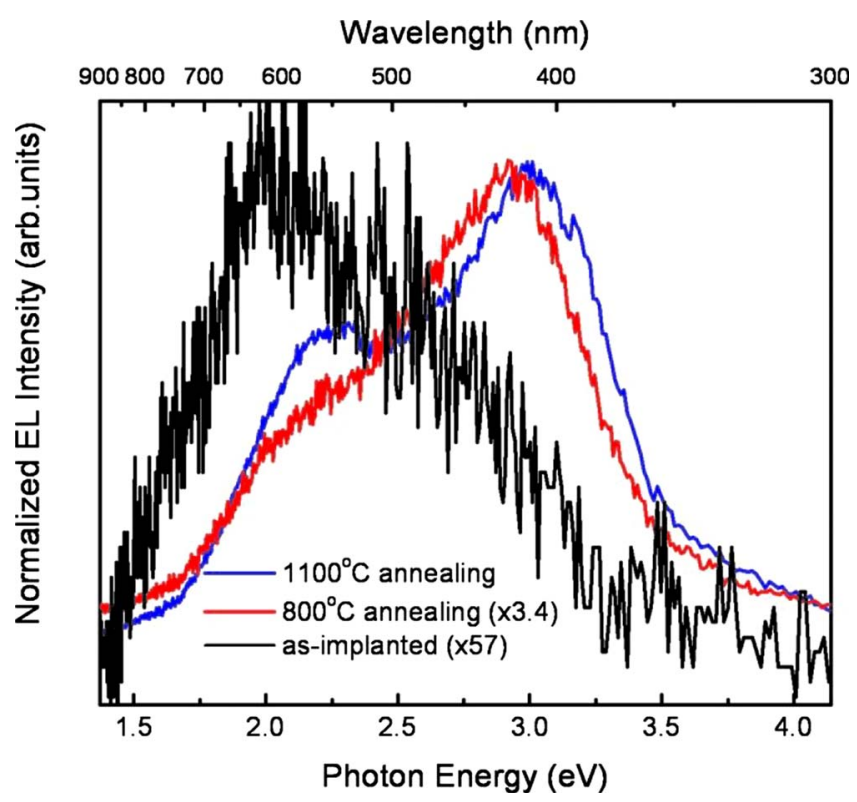

FIG. 4. (Color online) EL spectra for the as-implanted and annealed films under the gate voltage of $-17 \mathrm{~V}$.

Typical EL spectra at the gate voltage $\left(V_{g}\right)$ of $-17 \mathrm{~V}$ are shown in Fig. 4 for both the annealed films and the asimplanted film. In the EL spectrum of the as-implanted film, a broad peak located at an orange wavelength of about 600 $\mathrm{nm}(\sim 2.0 \mathrm{eV})$ was observed. While for the annealed film, a dominant peak at around $400 \mathrm{~nm}(\sim 3.1 \mathrm{eV})$ and a shoulder band at around $550 \mathrm{~nm}(\sim 2.3 \mathrm{eV})$, which make up a nearly white EL emission, dominate the EL spectra. The EL spectra can be deconvoluted into Gaussian-shaped EL bands. As an example, the EL spectral deconvolution for the $800{ }^{\circ} \mathrm{C}$ annealed film under the gate voltage of $-17 \mathrm{~V}$ is shown in Fig. 5(a). All the EL spectra of the as-implanted and annealed films consist of two major bands named as "shortwavelength major band" and "long-wavelength major band," respectively, in the present study. In addition, a minor ultraviolet (UV) band emerges for the annealing at both 800 and $1100{ }^{\circ} \mathrm{C}$, and one more minor near-IR band appears for the annealing at $1100{ }^{\circ} \mathrm{C}$, as shown in Fig. 5(b). ${ }^{11}$ Figure 6(a) shows the evolution of the center positions of the EL bands with annealing temperature under the gate voltage of $-17 \mathrm{~V}$. The intensity and the full width at half maximum (FWHM) of each EL band are shown as a function of the annealing temperature in Figs. 6(b) and 6(c), respectively.

For the as-implanted film, the short- and longwavelength major bands are located at $\sim 488 \mathrm{~nm}(2.54 \mathrm{eV})$ and $\sim 635 \mathrm{~nm}(1.95 \mathrm{eV})$, respectively. Annealing leads to blueshifts of the two major composite EL bands. For the annealing at $800{ }^{\circ} \mathrm{C}$, the short-wavelength major band shifts to $424 \mathrm{~nm}(2.92 \mathrm{eV})$ and the long-wavelength band shifts to $\sim 557 \mathrm{~nm}(2.22 \mathrm{eV})$. For the annealing at $1100{ }^{\circ} \mathrm{C}$, the short-wavelength major band slightly shifts to $411 \mathrm{~nm}(3.01$ $\mathrm{eV}$ ) further, while no significant shift occurs to the longwavelength major band. As the annealing temperature is increased from 800 to $1100{ }^{\circ} \mathrm{C}$, the UV band that becomes remarkable after annealing shifts from $334 \mathrm{~nm}(3.71 \mathrm{eV})$ to a
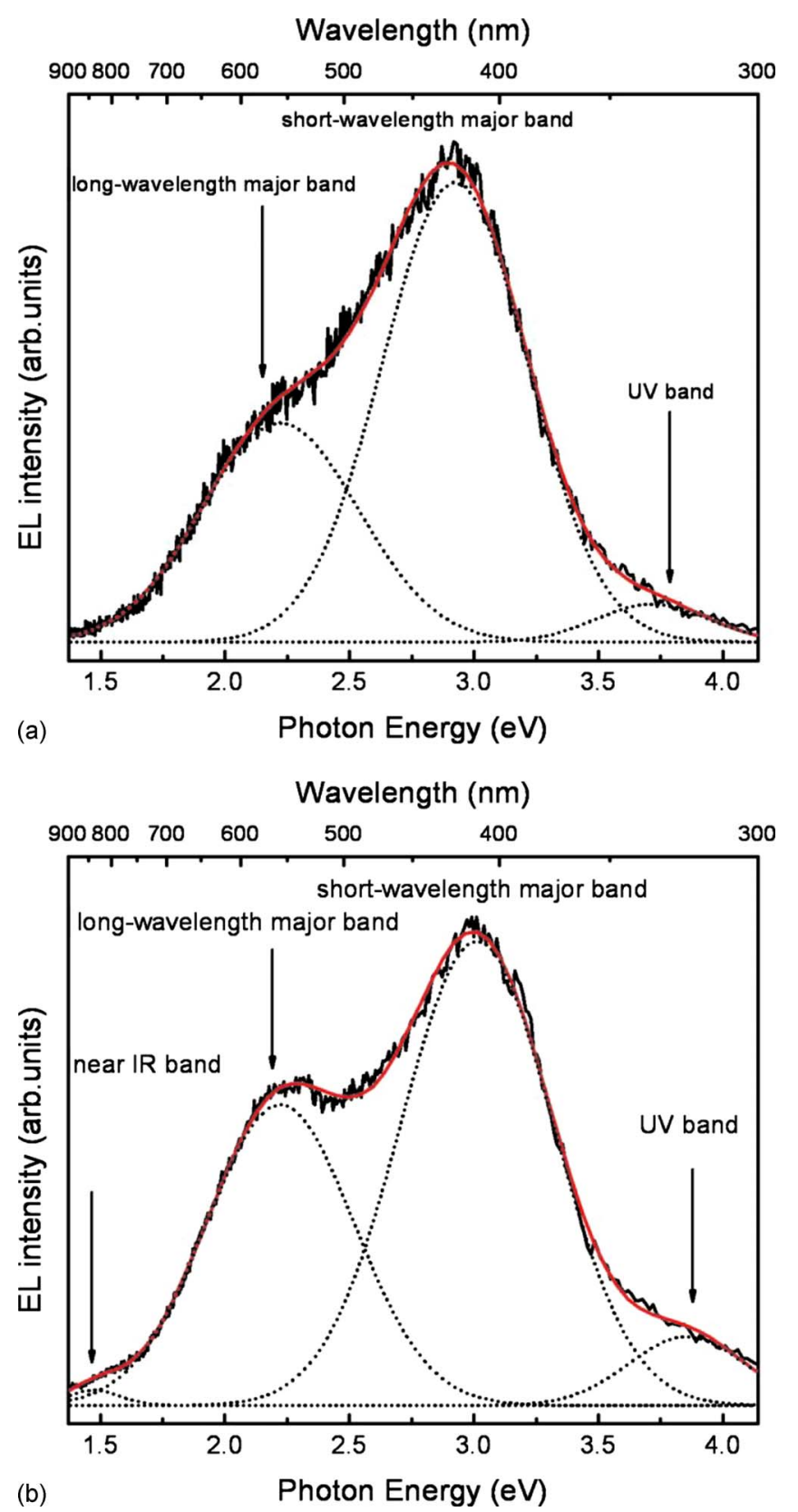

FIG. 5. (Color online) Deconvolution of EL spectrum at the gate voltage of $-17 \mathrm{~V}$ for the annealing at (a) $800{ }^{\circ} \mathrm{C}$ and (b) $1100{ }^{\circ} \mathrm{C}$.

shorter wavelength of $321 \mathrm{~nm}(3.86 \mathrm{eV})$ as well. The near IR band that is detectable after the annealing at $1100{ }^{\circ} \mathrm{C}$ is located at $842 \mathrm{~nm}(1.47 \mathrm{eV})$.

For the as-implanted film, the short- and longwavelength major bands have the same intensity. However, the FWHM of the short-wavelength major band is around $0.88 \mathrm{eV}$, larger than that of the long-wavelength major band $(0.49 \mathrm{eV})$. The contributions of both the short- and longwavelength major bands lead to an EL peak close to the long-wavelength major band. After the annealing, the intensity of the short-wavelength major band increases faster than the long-wavelength major band, and the short-wavelength major band becomes the dominant one. On the other hand, the FWHMs of the two major EL bands for the annealed films are about the same with the values of about $0.6 \mathrm{eV}$. Although the two additional EL bands (i.e., the UV and near 

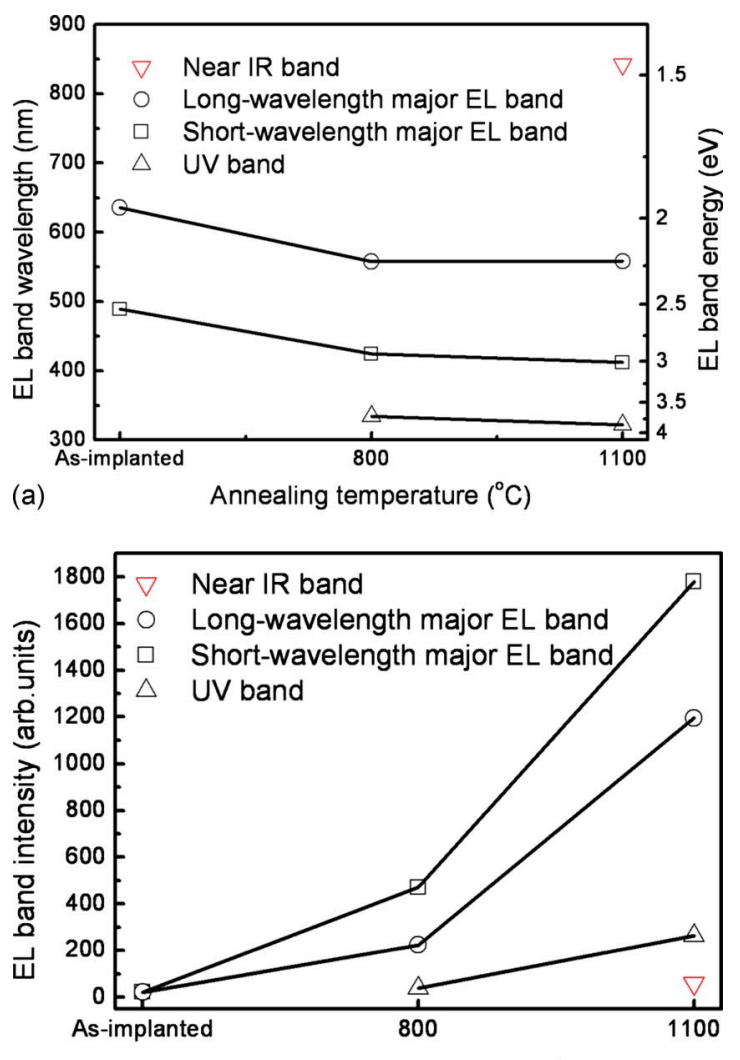

(b)

Annealing temperature $\left({ }^{\circ} \mathrm{C}\right)$

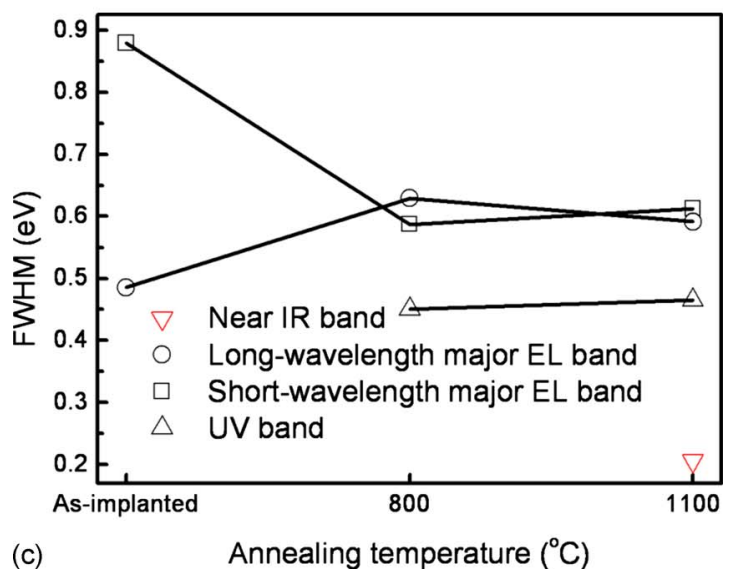

FIG. 6. (Color online) Evolution of each EL band with annealing temperature at the gate voltage of $-17 \mathrm{~V}$ : (a) the peak energy, (b) the intensity, and (c) the FWHM.

IR bands) emerge as the annealing temperature is increased, their intensities are much lower than that of the two major EL bands, as shown in Fig. 6(b). Despite its small contribution to the total EL intensity, the UV band becomes stronger for a higher annealing temperature.

For silicon nitride, it has been demonstrated by theoretical calculations and experimental results that states related to various defects, such as the silicon dangling bonds $\left(\equiv \mathrm{Si}^{0}\right)$, the nitrogen dangling bonds $\left(=\mathrm{N}^{-}\right)$and the $\equiv \mathrm{Si}-\mathrm{Si}$ $\equiv$ unit, exist in the band gap of silicon nitride. ${ }^{19-23}$ These defect states can involve in the radiative recombination of electrons and holes in silicon nitride, playing a role as luminescent centers. Due to the large amounts of $\equiv \mathrm{Si}-\mathrm{Si} \equiv$ bonding states $(\sigma)$ and $=\mathrm{N}^{-}$states, a wide valence band tail

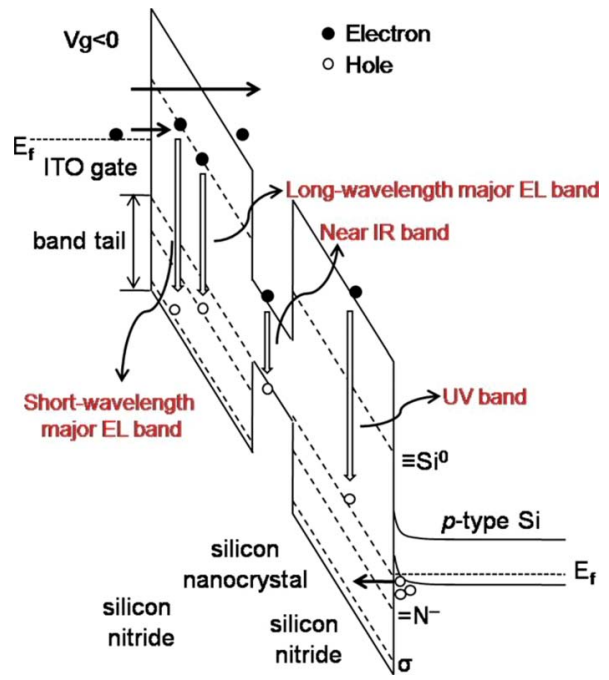

FIG. 7. (Color online) Schematic band diagram of the LED structure under forward bias. Carrier transport, defect states, and $\mathrm{Si}$ nanocrystal in the nitride film and various recombination processes are illustrated in the diagram also.

with the width of $1.5 \mathrm{eV}$ could exist in the SRN, ${ }^{20,21}$ particularly in the Si-implanted silicon nitride film with high structural disorder and many point defects. The wide valence band tail can assist in providing holes for radiative recombination.

The two distinct major bands of the annealed films suggest two different luminescent mechanisms. The shortwavelength major band at around $3.0 \mathrm{eV}$ can be attributed to the radiative transitions from the defect state related to $\equiv \mathrm{Si}^{0}$, which is located at $3.1 \mathrm{eV}$ above the valence band maximum and acts as electron traps, ${ }^{20,22}$ to the valence band or the band tail states close to the valence band, such as the bonding state $(\sigma)$ of $\equiv \mathrm{Si}-\mathrm{Si} \equiv .^{19-21}$ On the other hand, the longwavelength major band with the center energy of $\sim 2.2 \mathrm{eV}$ observed in this study could be due to the electronic transitions between the $\equiv \mathrm{Si}^{0}$ luminescent center and the hole trap state of $=\mathrm{N}^{-}$in the valence band tail, which is located at about $0.8 \mathrm{eV}$ above the valence band maximum. ${ }^{19,22,23}$ The radiative recombination processes for the major EL bands are schematically shown in Fig. 7.

Besides the annealed film, the EL of the as-implanted film also consist of two major bands, and the EL spectrum is similar to those reported for the plasma enhanced chemicalvapor deposition (PECVD) SRN films without postdeposition annealing. ${ }^{21,24}$ The EL band positions of asdeposited PECVD SRN films reported in Ref. 24 are around 500 and $620 \mathrm{~nm}$, being close to the major band positions of the as-implanted film in this study (i.e., 488 and $635 \mathrm{~nm}$ ). Based on the analysis of Refs. 21 and 24, the EL of the as-implanted film can be also explained by the radiative recombination from the luminescent centers such as $\equiv \mathrm{Si}^{0}$ to different states in the valence band tail, being similar to the mechanisms proposed for the annealed films. Blueshifts of the two major EL bands with annealing temperature, as shown in Fig. 6(a), may be originated from the evolution of the band tail states with annealing. During the annealing, the implantation-induced damage can be recovered and the $\mathrm{Si}$ - 
implanted film can evolve toward an ordered state. As a consequence, the band tail is reduced and becomes shallower from the band edge. Thus, the recombination from the luminescent centers to the states in the reduced band tail can produce light emissions with higher energies.

When the Si-implanted films are annealed, two minor EL bands, i.e., the UV band and the near IR band emerge. As shown in Fig. 7, under a high gate voltage, electrons can inject into the conduction band of silicon nitride and then recombine with the holes in the band tail above the silicon nitride valence band, leading to the UV band emission. As can be seen in Fig. 6(a), the UV band blueshifts as the annealing temperature is increased from 800 to $1100{ }^{\circ} \mathrm{C}$, being similar to the situation of the short-wavelength major band. This shift can also result from the reduced valence band tail at the higher annealing temperature. Regarding the near IR band which can be detected for the annealing at $1100{ }^{\circ} \mathrm{C}$, it is believed to be related to the light emission from the $\mathrm{Si}$ nanocrystals formed during the post-implantation annealing at the high temperature. ${ }^{3,17}$ Due to the quantum confinement effect, the band gap of the Si nanocrystals is expanded as compared with bulk $\mathrm{Si}^{25}$ As shown in Fig. 7, the recombination of the injected electrons and holes in the conduction and valence bands of the Si nanocrystals, respectively, can produce the near IR band emission if the bandgap of the nanocrystals is about $1.4 \mathrm{eV}$.

\section{CONCLUSION}

In summary, the effect of postimplantation annealing on the performance of the LED based on multiple Si-implanted silicon nitride film has been investigated. The increasing of annealing temperature causes a reduction in the injection current but enhances the EL intensity significantly. As a result, the efficiency of the LEDs is improved by the annealing at a high temperature. Moreover, the composite EL bands blueshift as the annealing temperature is increased, which has been explained in terms of band tail states.

\section{ACKNOWLEDGMENTS}

This work has been financially supported by the National Research Foundation of Singapore (Gant No. NRF-G-CRP
2007-01). Y. Liu would like to acknowledge NSFC under Grant No. 60806040.

${ }^{1}$ L. Pavesi, L. Dal Negro, C. Mazzoleni, G. Franzò, and F. Priolo, Nature (London) 408, 440 (2000).

${ }^{2}$ G.-R. Lin, C. J. Lin, C. K. Lin, L. J. Chou, and Y. L. Chueh, J. Appl. Phys. 97, 094306 (2005).

${ }^{3}$ L. Ding, T. P. Chen, Y. Liu, M. Yang, J. I. Wong, K. Y. Liu, F. R. Zhu, and S. Fung, Nanotechnology 18, 455306 (2007).

${ }^{4}$ N.-M. Park, C.-J. Choi, T.-Y. Seong, and S.-J. Park, Phys. Rev. Lett. 86, 1355 (2001)

${ }^{5}$ L. Dal Negro, J. H. Yi, L. C. Kimerling, S. Hamel, A. Williamson, and G. Galli, Appl. Phys. Lett. 88, 183103 (2006).

${ }^{6}$ M. Makarova, J. Vuckovic, H. Sanda, and Y. Nishi, Appl. Phys. Lett. 89, 221101 (2006).

${ }^{7}$ M. Wang, M. Xie, L. Ferraioli, Z. Yuan, D. Li, D. Yang, and L. Pavesi, J. Appl. Phys. 104, 083504 (2008).

${ }^{8}$ L.-Y. Chen, W.-H. Chen, and F. C.-N. Hong, Appl. Phys. Lett. 86, 193506 (2005).

${ }^{9}$ R. Huang, K. J. Chen, P. G. Han, H. P. Dong, X. Wang, D. Y. Chen, W. Li, J. Xu, Z. Y. Ma, and X. F. Huang, Appl. Phys. Lett. 90, 093515 (2007).

${ }^{10}$ J. Warga, R. Li, S. N. Basu, and L. Dal Negro, Appl. Phys. Lett. 93, 151116 (2008).

${ }^{11}$ Z. H. Cen, T. P. Chen, L. Ding, Y. Liu, J. I. Wong, M. Yang, Z. Liu, W. P. Goh, F. R. Zhu, and S. Fung, Appl. Phys. Lett. 94, 041102 (2009).

${ }^{12}$ X. Yu, L. Shao, B. Dharmaiahgari, X. Wang, H. Chen, Q. Y. Chen, K. Ma, J. Liu, and W.-K. Chu, Nucl. Instrum. Methods Phys. Res. B 249, 414 (2006).

${ }^{13}$ H. E. Romero and M. Drndic, Phys. Rev. Lett. 95, 156801 (2005).

${ }^{14}$ Y. Liu, T. P. Chen, C. Y. Ng, M. S. Tse, S. Fung, Y. C. Liu, S. Li, and P. Zhao, Electrochem. Solid-State Lett. 7, G134 (2004).

${ }^{15}$ T. P. Chen, M. S. Tse, and X. Zeng, Appl. Phys. Lett. 78, 492 (2001).

${ }^{16}$ A. R. Wilkinson and R. G. Elliman, J. Appl. Phys. 96, 4018 (2004).

${ }^{17}$ M. H. Wang, D. S. Li, Z. Z. Yuan, D. R. Yang, and D. L. Que, Appl. Phys. Lett. 90, 131903 (2007).

${ }^{18}$ S. V. Deshpande, E. Gulari, S. W. Brown, and S. C. Rand, J. Appl. Phys. 77, 6534 (1995)

${ }^{19}$ H. L. Hao, L. K. Wu, W. Z. Shen, and F. W. Dekkers, Appl. Phys. Lett. 91, 201922 (2007).

${ }^{20}$ J. Robertson and M. J. Powell, Appl. Phys. Lett. 44, 415 (1984).

${ }^{21}$ M. Wang, J. Huang, Z. Yuan, A. Anopchenko, D. Li, D. Yang, and L. Pavesi, J. Appl. Phys. 104, 083505 (2008).

${ }^{22}$ C. M. Mo, L. D. Zhang, C. Y. Xie, and T. Wang, J. Appl. Phys. 73, 5185 (1993).

${ }^{23}$ Z. Pei, Y. R. Chang, and H. L. Hwang, Appl. Phys. Lett. 80, 2839 (2002).

${ }^{24}$ R. Huang, H. P. Dong, D. Q. Wang, K. J. Chen, H. L. Ding, X. Wang, W. Li, J. Xu, and Z. Y. Ma, Appl. Phys. Lett. 92, 181106 (2008).

${ }^{25}$ M. V. Wolkin, J. Jorne, P. M. Fauchet, G. Allan, and C. Delerue, Phys. Rev. Lett. 82, 197 (1999). 